\title{
Frequency-Dependent Tuning of the Human Motor System Induced by Transcranial Oscillatory Potentials
}

\author{
Matteo Feurra, Giovanni Bianco, Emiliano Santarnecchi, Massimiliano Del Testa, Alessandro Rossi, and Simone Rossi \\ Dipartimento di Neuroscienze, Sezione Neurologia e Neurofisiologia Clinica, Azienda Ospedaliera-Universitaria Senese, Policlinico Le Scotte, I-53100 Siena, \\ Italy
}

Different corticothalamic brain modules intrinsically oscillate at a "natural frequency" in a topographically organized manner. In "quiescent" human sensorimotor regions, the main detectable oscillatory activity peaks at $\sim 20 \mathrm{~Hz}$, and partly contributes to determine the state of corticospinal excitability. Here, we showed that the transcranial application of an imperceptible, short-lasting ( $90 \mathrm{~s})$ electric field oscillating at a physiological range increases corticospinal excitability online, with well defined frequency dependence and regional specificity. Indeed, the size of motor evoked potentials (MEPs) induced by navigated single-pulse TMS over the motor cortex significantly increased only during the local application of transcranial alternating current stimulation (tACS) at $20 \mathrm{~Hz}$ ( $\beta$ range). Other tACS frequencies $(5,10$, and $40 \mathrm{~Hz})$ applied on the motor cortex did not impact MEPs' size. Moreover, tACS applied on a control site (parietal cortex) and on a peripheral site (ulnar nerve) also failed to modulate MEPs. These results help clarifying the functional significance of the $20 \mathrm{~Hz}$ idling $\beta$ rhythm of sensorimotor regions and suggest potential clinical applications of this approach.

\section{Introduction}

A fundamental and phylogenetically preserved property of neurons is their ability to oscillate within a wide range of rhythmic activity, from 0.05 to $500-600 \mathrm{~Hz}$ (Buzsáki and Draguhn, 2004). Oscillatory activity is recorded on the scalp with electroencephalography (EEG), and it has been interpreted as the net result of spontaneous, physiological variations of cortical, subcortical, and spinal motoneuronal excitability around a fluctuating critical firing level, known as the "uncertain range" (Adrian and Moruzzi, 1939; Liddell and Phillips, 1952; Henneman et al., 1974).

Rhythmic oscillations get organized according to the main states of the brain such as sleep (low frequencies) or resting wake (high-frequency bands). More complex regional patterns of oscillatory activities can take place according to the behavioral tasks on which the brain is currently engaged, thereby conveying informational relevant contents (Thut and Miniussi, 2009). Cognitive processes like memory, perception, or even dreaming and consciousness, could result from the combination of regional or diffuse synchronization of neural assemblies in a temporally specific manner (Gray et al., 1989; Llinás and Ribary, 1993; Engel et al., 2001; Varela et al., 2001).

In human sensorimotor regions, the main detectable oscillatory activity is an "idling" beta activity peaking at $\sim 20 \mathrm{~Hz}(\beta$

Received Feb. 23, 2011; revised May 19, 2011; accepted May 23, 2011.

Author contributions: M.F. and S.R. designed research;M.F., G.B., E.S., M.D.T., and S.R. performed research; M.F., G.B., and E.S. analyzed data; M.F., A.R., and S.R. wrote the paper.

This work has been partially supported by the European Commission with the Collaborative Project No. 248587, "THE Hand Embodied," within the FP7-ICT-2009-4-2-1 program "Cognitive Systems and Robotics." We thank Drs. Filippo Giorgi, Federica Ginanneschi, and Caterina Marotti for experimental support.

Correspondence should be addressed to Dr. Simone Rossi, Dipartimento di Neuroscienze, Sezione Neurologia e Neurofisiologia Clinica, Azienda Ospedaliera-Universitaria Senese, Policlinico Le Scotte, Viale Bracci, I-53100 Siena, Italy. E-mail: Rossisimo@unisi.it.

DOI:10.1523/JNEUROSCI.0978-11.2011

Copyright $\odot 2011$ the authors $\quad 0270-6474 / 11 / 3112165-06 \$ 15.00 / 0$ range), which typically occurs in the absence of processing sensory information or motor output (Niedermeyer, 1999). Such an activity is usually associated with spontaneous oscillations in the alpha band $(8-12 \mathrm{~Hz})$, known as mu rhythm (Gastaut, 1952; Kuhlman, 1978; Sauseng et al., 2009). However, the mu rhythm includes a wider class of rhythms, which are usually not sinusoidal (Pfurtscheller et al., 1997). Moreover, they have different topography and frequency (McFarland et al., 2000). Several studies reported that mu rhythm may be attenuated or desynchronized by imaging, preparation, and execution of a movement (Kuhlman, 1978; Pfurtscheller, 1989, 1998; Niedermeyer, 1997).

Solid evidence indicates that also $\beta$ oscillations of sensorimotor areas usually undergo desynchronization during preparation, execution, imagination, or observation of movements (Jasper and Penfield, 1949; Chatrian et al., 1959; Pfurtscheller and Aranibar, 1977; Salmelin and Hari, 1994; Rossi et al., 2002).

On the other hand, EEG disclosed $\beta$-range synchronization between the oscillatory activity recorded over motor areas and the electromyographic (EMG) activity of contralateral muscles engaged in static low-level force production tasks both in the monkey and in the human motor system (Sanes and Donoghue, 1993; Kilner et al., 1999; Salenius and Hari, 2003; Kristeva et al., 2007). This synchronization probably underpins the integration of online sensorimotor processes required to maintain a constant motor output (Baker, 2007). Therefore, the causal relationships between the $\beta$ idling oscillatory activity of the motor areas and the related human corticospinal output still need to be clarified.

To this aim, we used a novel approach that combines simultaneous single-pulse transcranial magnetic stimulation (TMS) and transcranial alternating current stimulation (tACS) (Antal et al., 2008) applied at different frequencies. The rationale for the combined use of the two techniques lies on the different information that they convey. TMS transsynaptically activates fast- 
conducting pyramidal corticospinal axons (Zarola et al., 1989; Di Lazzaro et al., 2003), and therefore mostly reflects the physiological properties of human motor pathways (Hallett, 2000; Rossini and Rossi, 2007). tACS instead is supposed to entrain regional brain oscillations in a frequency-dependent manner, thereby interacting with specific functions of the stimulated region (Kanai et al., 2008, 2010; Pogosyan et al., 2009; Thut and Miniussi, 2009; Paulus, 2010).

To examine the frequency dependence and the spatial specificity of tACS effects, seven conditions were included in a randomized blocked design. For each experimental conditions, corticospinal excitability was assessed by the average amplitude of 10 single-pulse neuronavigated TMS pulses on subjects at rest. First, a basal session was run (without tACS conditioning). Then, tACS was applied over the left motor cortex at 5 $\mathrm{Hz}$ ( $\theta$ band), $10 \mathrm{~Hz}$ ( $\alpha$ band), $20 \mathrm{~Hz}$ ( $\beta$ band), and $40 \mathrm{~Hz}$ ( $\gamma$ band). tACS was also applied at $20 \mathrm{~Hz}$ over the right parietal cortex to control for unspecific effects on cortical excitability. The experiment also included an additional basal session to check for the stability of nonconditioned MEPs and to rule out carry-over effects of tACS (Fig. 1a).

\section{Materials and Methods}

Participants. Fifteen healthy volunteers ( 7 females, 8 males; mean age $33.3 \pm 8.8$ years), with normal neurological examination, were included in the study. All participants reported to be fully right-handed, and were naive to the purpose of the experiment. None of them reported the use of drugs or alcohol in the days preceding the experiment. All subjects gave written informed consent. The study was approved by the local Ethics Committee. Subjects sat comfortably in a reclining chair. They kept their arm fully relaxed in a natural position, and their hands pronated, resting on a pillow.

Stimulating and recording procedures. Single-pulse TMS was performed via a standard eight-shaped focal coil (diameter of each wing 70 $\mathrm{mm}$ ) connected with a monophasic Bistim 200 stimulator with $\sim 2.2$ tesla as maximal output (MagStim). The coil was positioned on the left hemiscalp, with the handle pointing backwards and at $\sim 45^{\circ}$ from the midline. The "hot spot" was the scalp location eliciting MEPs of $<50 \mu \mathrm{V}$ with $50 \%$ probability, corresponding to the resting motor threshold (Rossi et al., 2009). This position was marked on the scalp with a pencil.

A navigated stimulation system (SofTaxic optically tracked by EMS) was used throughout the experiment (Fig. 1b). This system allowed the exact repositioning of the TMS coil within and across experimental sessions. The software uses passive spherical markers applied both on the coil and on the subjects' head. Marker positions were recorded by an optical digitizer (Polaris Vicra, NDI) and reproduced on the computer screen. The system detects any difference in spatial coil location and orientation (three rotation angles) with respect to the initial pulse, with a tolerance of $<2 \mathrm{~mm}$ for each dimension. This procedure thus provides three-dimensional online information on the initial and actual coil placements, while minimizing the variability of TMS-induced electric fields directly measured within a scalp model (Cincotta et al., 2010).

tACS. tACS was delivered through a constant current stimulator charged with a battery (Eldith DC-Stimulator by Neuro Conn). The tACS stimulator was connected to conductive-rubber electrodes (size $5 \times 7 \mathrm{~cm}$ ) placed in sponges and applied on the scalp. To minimize skin sensation, the electrodes were constantly saturated with a saline solution, and impedances were kept below $10 \mathrm{k} \Omega$ throughout stimulation sessions.

The "target" electrode was centered on the scalp overlying the hot spot of the left motor cortex as determined by TMS, whereas the other stimulating electrode ("reference") was placed on $\mathrm{Pz}$ according to the International 10-20 EEG System. An additional sponge electrode was placed on the right parietal cortex (PC), corresponding to P4 of the International 10-20 EEG System. This was used as a control site of stimulation. Rubber strips around the head guaranteed stable electrode-scalp contact for the three electrodes. Several electrode montages have been suggested to make tACS more focal (Datta et al., 2009; Bikson et al., 2010). The electrode positioning used here was based on previous studies in which clear frequency-specific effects of tACS were found (Kanai et al., 2010; Feurra et al., 2011).

A sinusoidal stimulation with no DC offset was delivered at an intensity of $1000 \mu \mathrm{A}$ (peak-to-peak). This low intensity of stimulation was chosen to avoid the perception of flickering lights usually reported by subjects with higher stimulation intensities (Kanai et al., 2008), and supposed to be originated at a retinal level, due to the electric fields volume conduction toward the scalp sites with lower resistance, such as the orbit (Paulus, 2010). The average current density at the stimulation electrode was $\sim 14.2 \mu \mathrm{A} / \mathrm{cm}^{2}$. However, it should be noted that the peak current density that occurs near the edges of the electrodes is much higher due to the "edge effect" (Miranda et al., 2006, 2009).

Seven different conditions were run: a basal 1 session (without tACS), followed by tACS on the left motor cortex at $5 \mathrm{~Hz}(\theta$ band), $10 \mathrm{~Hz}(\alpha$ band), $20 \mathrm{~Hz}$ ( $\beta$ band), and $40 \mathrm{~Hz}$ ( $\gamma$ band). A $20 \mathrm{~Hz}$ tACS application on the right parietal cortex was used as control for unspecific effects on cortical excitability. A second basal session (basal 2) was then delivered to check for the stability of nonconditioned MEPs and for conditioning aftereffects. The order of tACS conditions was fully randomized and counterbalanced between subjects. Each stimulation session lasted no more than $90 \mathrm{~s}$ (Fig. 1a). The interstimulus interval (ISI) was twice the length of each tACS application. This ISI was chosen to avoid carry-over effects of tACS. It is also worth noting that in previous studies, no after- 
effects of tACS at $20 \mathrm{~Hz}$ on the motor (Moliadze et al., 2010) or on the visual cortex (Antal et al., 2008; Kanai et al., 2010) emerged. Due to the low and gentle rise of the intensity of stimulation, subjects did not feel any scalp sensation. At debriefing, subjects reported that they were blind to the frequency applied and that they were not aware of the location of the stimulation (i.e., whether tACS was applied on the motor or on the parietal cortex). Only 4 of 15 subjects reported slight flickering sensations in their peripheral visual field.

TMS. During tACS in each experimental session, TMS was applied over the sponge electrode overlying the left motor cortex (Fig. 1b). MEPs were recorded from the right first dorsal interosseus (FDI). As the rising time to reach the determined intensity of stimulation was $\sim 5 \mathrm{~s}$, the start of MEPs recording was not earlier than $20 \mathrm{~s}$ of stimulation with tACS. The TMS intensity was adjusted to produce an amplitude of $600-800 \mu \mathrm{V}$ in basal conditions (i.e., without tACS, but while TMS was applied on the electrode overlying the left motor cortex). $\mathrm{Ag}-\mathrm{AgCl}$ adhesive electrodes were positioned over the muscle with the active electrode on its motor point and the negative on the nearest finger joint.

Ten MEPs/condition, spaced at least $7 \mathrm{~s}$, were recorded by a fourchannel electromyograph (Phasis, EBNeuro), with a bandpass filter of 20 $\mathrm{Hz}$ to $5 \mathrm{kHz}$, sampled at $20 \mathrm{kHz}$, with an acquisition sensitivity ranging from 0.1 to $1 \mathrm{mV}$. MEPs were discarded from postprocessing if an EMG burst preceded the TMS by 300 to $0 \mathrm{~ms}$ or if an MEP-to-MEP latency jitter of $>2$ ms appeared. Both these events could potentially signal unwanted muscular activation, and therefore a bias of the MEP amplitude.

The mean peak-to-peak amplitude values of $10 \mathrm{MEPs}$ from the right FDI muscle (obtained by navigated TMS of the left motor cortex over the tACS sponge electrode) (Fig. 1b) were calculated for each experimental condition.

Peripheral control. To rule out a physical interaction between the electric fields induced by single-pulse TMS and those produced by tACS, the same interleaved TMS/tACS protocol was repeated on the right forearm in 12 subjects. The "target" and the "reference" electrodes were placed on the skin overlying the ulnar nerve at the elbow and on the volar surface of the forearm, respectively. tACS at $5 \mathrm{~Hz}$ and $20 \mathrm{~Hz}$, plus a basal condition (no-tACS), was applied with same parameters of the main experiment. During tACS, 10 compound muscle action potentials (CMAPs) from the right FDI muscle were recorded for each condition, after having placed the stimulating TMS coil over the target stimulating electrode, and adjusting the intensity of the stimulator to produce MEPs of similar size to those elicited by TMS over the scalp. Finally, an additional session without tACS was run, to prove the stability of MEPs size.

Subjects did not report skin sensations at debriefing and were not able to disentangle whether they were stimulated by tACS or not.

Data analysis. Postprocessing of data was performed in a blind manner with respect to the experimental conditions. MEPs amplitudes between the two largest peaks of opposite polarity were measured for each experimental session. Each amplitude value was transformed into natural logarithms (Nielsen, 1996). This procedure was used to normalize the distribution of amplitude data by reducing the heteroschedasticity between different conditions, therefore allowing interindividual comparisons. Amplitude data were then averaged for each condition. To get an optimal control over the results of the main experiment, a repeatedmeasure ANOVA was performed separately for the two basal conditions, the main experiment and the peripheral control experiment. Greenhouse-Geisser correction was applied when necessary to compensate for the violation of the assumption of sphericity. In the presence of significant interactions, corrected pairwise comparisons were performed by Bonferroni test. The level of significance was set at $p=0.05$.

\section{Results}

Three separate ANOVAs were performed to address the differences between the two basal conditions, the main experiment conditions, and the peripheral control experiment conditions.

\section{Basal conditions (no tACS)}

The ANOVA contrasting the MEP amplitude of the two basal conditions failed to show significant differences $\left[F_{(1,14)}=2.976\right.$,

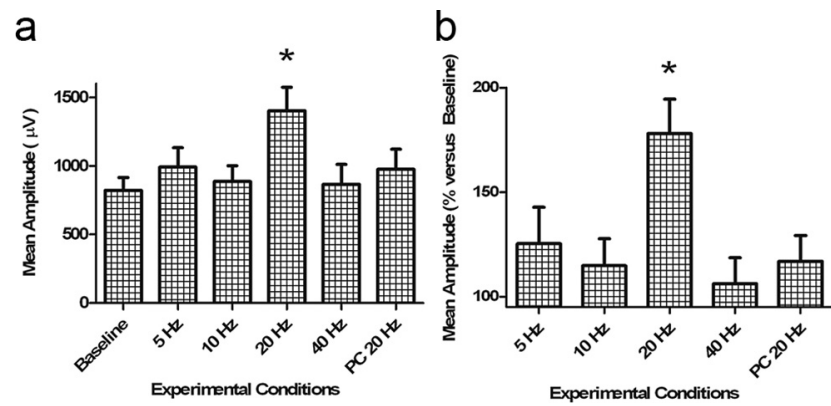

Figure 2. Results of the main experiment. $\boldsymbol{a}$, Average MEP amplitude ( $\pm \mathrm{SE}$ ) values (raw data) obtained through different experimental conditions. Note that only tACS delivered at $\beta$ range $(20 \mathrm{~Hz})$ on the motor cortex increases the corticospinal output versus all the other conditions (baseline, $5 \mathrm{~Hz}, 10 \mathrm{~Hz}, 40 \mathrm{~Hz}$, and $20 \mathrm{~Hz}$ on the parietal cortex). An asterisk $\left({ }^{*}\right)$ indicates a significant difference of $20 \mathrm{~Hz}$ stimulation with respect to all the other conditions. Statistics refer to log-transformed amplitude data (see Results). $\boldsymbol{b}$, Percentage changes versus baseline of MEP amplitude values (raw data).

mean square error $(\mathrm{MSE})=1.19, p=0.107]$ of mean natural logarithm (LN)-transformed MEP amplitude values (raw values: basal 1, 800.50 $\pm 101.2 \mu \mathrm{V}, \mathrm{LN}=6.59$; basal 2, 843.12 \pm 122.9, $\mathrm{LN}=6.58)$. This suggests that tACS did not produce any detectable aftereffects on corticospinal excitability and that at the end of the experiment the intrinsic excitability of the motor system was unchanged.

\section{Main experiment}

Following from the results of the previous analysis, data from the two basal conditions were collapsed into a new "baseline" condition. A one-way ANOVA was then performed to verify the occurrence of any frequency-dependent and regional-dependent effect of tACS on the corticospinal excitability of the left motor cortex. The ANOVA contrasted six levels of the factor "experimental condition" [baseline, tACS of the left motor cortex at $5 \mathrm{~Hz}(\theta$ range), $10 \mathrm{~Hz}$ ( $\alpha$ range), $20 \mathrm{~Hz}$ ( $\beta$ range), $40 \mathrm{~Hz}$ ( $\gamma$ range), and tACS on the right parietal cortex at $\beta$ range]. A significant main effect of experimental condition emerged $\left(F_{(5,70)}=4.362\right.$, MSE $=0.603, p=0.002$ ). Post hoc comparisons (Fig. 2) revealed a significant and selective effect of tACS on the left motor cortex at $20 \mathrm{~Hz}(\beta)$ (Fig. $2 a$ ). tACS at $20 \mathrm{~Hz}$ consistently enhanced the corticospinal output compared to all other experimental conditions $(20 \mathrm{~Hz}$ vs $5 \mathrm{~Hz}, p=0.016$; vs $10 \mathrm{~Hz}, p=0.045$; vs $40 \mathrm{~Hz}$, $p=0.039$; vs right $\mathrm{PC} 20 \mathrm{~Hz}, p=0.031$, vs baseline, $p=0.002$ ). All other pairwise comparisons were not significant. Focusing on individual responses, 11 out 15 subjects showed the highest MEPs during tACS at the $\beta$ range. Among the remaining four subjects, tACS at $10 \mathrm{~Hz}$ induced the highest facilitation in two, $40 \mathrm{~Hz}$ in one, and $5 \mathrm{~Hz}$ in another one. In these four subjects, the $20 \mathrm{~Hz}$ tACS was "the second-best frequency" in terms of MEP facilitation.

MEPs latency did not change within and across conditions. To better illustrate the $20 \mathrm{~Hz}$ tACS effect, percentage changes versus baseline of log-transformed MEP amplitude values, average of MEP amplitude values (raw data), and percentage changes versus baseline of MEP amplitude values (raw data) are shown in Figure $2, a$ and $b$.

\section{Peripheral control experiment}

The control experiment aimed to rule out a physical interaction between the electric fields induced by tACS and the current flow generated by single-pulse TMS. The same interleaved TMS/tACS protocol was repeated in 12 subjects on the right forearm (ulnar 


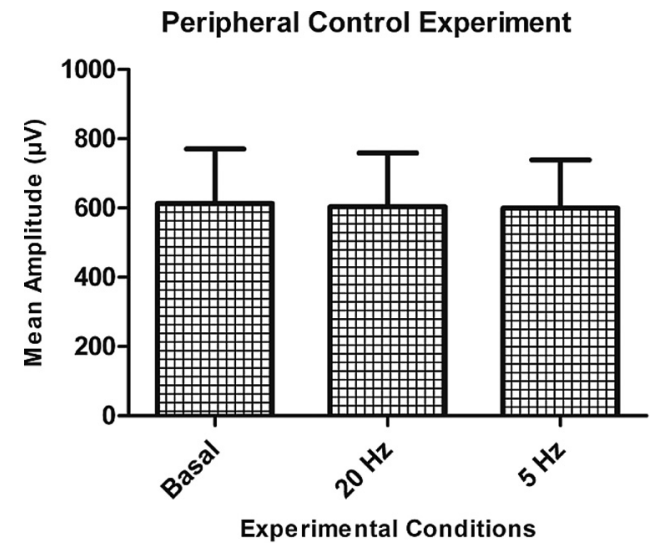

Figure 3. Results of the control peripheral experiment. The three columns indicate the average amplitude ( $\pm \mathrm{SE}$ ) values (raw data) of the CMAPs obtained by TMS on the ulnar nerve at the elbow during local tACS at $20 \mathrm{~Hz}$ and at $5 \mathrm{~Hz}$ versus baseline (i.e., no $\mathrm{tACS}$ ). No changes are observed. Results of statistical tests, performed with log-transformed amplitude data, are in the Results section.

nerve), thus bypassing the transsynaptic activation of pyramidal neurons commonly induced by TMS.

Analysis of the ulnar nerve stimulation data was performed with a one-way ANOVA contrasting three levels of the factor "experimental condition" (basal, $5 \mathrm{~Hz}, 20 \mathrm{~Hz}$ condition). No significant effect emerged ( $p=0.480$; raw values were $613 \pm$ $523.03 \mu \mathrm{V}, \mathrm{LN}=6.42$ for basal, $603.92 \pm 512.84 \mu \mathrm{V}, \mathrm{LN}=6.40$ for $5 \mathrm{~Hz}$, and $599.73 \pm 460.50 \mu \mathrm{V}, \mathrm{LN}=6.40$ for $20 \mathrm{~Hz}$ ). This suggests that tACS frequencies did impact the amplitude of CMAPs evoked by magnetic stimulation of the ulnar nerve. It is therefore unlikely that the results of the main experiment are confounded by an interaction between the TMS and tACS electric fields (Fig. 3).

\section{Discussion}

The EEG of normal waking adults suggests that different corticothalamic brain modules are tuned to oscillate at a topographically organized "natural frequency" (Rosanova et al., 2009). In sensorimotor areas, this natural frequency is mainly represented by an "idling" $\beta$ activity peaking at $\sim 20 \mathrm{~Hz}$, which typically occurs in the absence of sensory information processing or motor output (Salmelin and Hari, 1994; Niedermeyer, 1999).

The current results originally provide the evidence of a causal role of rolandic $20 \mathrm{~Hz}$ rhythm in facilitating corticospinal neurons, at least under the current "quiescent" experimental circumstances. The overall magnitude of the output volley evoked by a fixed test stimulus increased only when a conditioning $20 \mathrm{~Hz}$ oscillating current was applied on the scalp overlying the motor cortex. This effect was frequency dependent, as it occurred only when the frequency of tACS $(20 \mathrm{~Hz})$ overlapped with the prevailing natural frequency (i.e., $\beta$ range) of motor areas at rest. Indeed, tACS delivered at the $\theta, \alpha$, and $\gamma$ range did not change the amplitude of corticospinal response with respect to baseline. This effect was also anatomically specific: $\beta$ frequency applied on the right parietal cortex did not affect the corticospinal output, despite the existence of strong anatomical and functional ipsilateral and contralateral parietomotor connections (Rizzolatti and Luppino, 2001; Koch et al., 2009).

The lack of modulation of corticospinal output during tACS at alpha band may suggest that mu rhythm is less susceptible to tACS-induced "entrainment" phenomena (Thut and Miniussi, 2009). This could be related to the intrinsic characteristics of the mu rhythm. Compared to the typical occipital alpha rhythm, mu rhythm is usually not sinusoidal (Pfurtscheller et al., 1997) and is topographically best represented in postcentral regions, rather than in the motor cortex (McFarland et al., 2000).

The results of the peripheral control experiment allowed us to rule out unspecific biophysical interactions between the electric fields induced by tACS and those induced by TMS pulses, which might have interfered with the physiological modulations of MEPs. Variability of MEPs amplitude due to small displacements of the coil can also be excluded thanks to the online neuronavigation procedure. Peripheral control of tACS effects and TMS neuronavigation have never been used in previous studies addressing similar questions (Kanai et al., 2008; Terney et al., 2008; Moliadze et al., 2010). Regional specificity has been investigated only in one study on the visual system (Kanai et al., 2010). Therefore, it is reasonable to conclude that the increase in MEP size was due to a frequency-dependent facilitation of corticospinal neurons.

These findings are particularly striking considering that they are obtained within 90 s of tACS per condition. Such an immediate effect on corticospinal excitability was lacking when tACS had been delivered at ripple range (i.e., $>80-100 \mathrm{~Hz}$ ) (Moliadze et al., 2010). This is probably because the physiological weight of the $20 \mathrm{~Hz}$ rhythm in sensorimotor regions is definitely higher than the one of the ripple frequencies, which are usually observed as short-lived bursts at hippocampal but not sensorimotor regions (Buzsáki and Draguhn, 2004; Epsztein et al., 2010). Future studies should compare the effects of tACS applications of similar duration, both at $\beta$ and at ripple ranges [ $10 \mathrm{~min}$ in the study by Moliadze et al. (2010)].

The present findings nicely integrate previous evidence on the effects of tACS delivered over other neocortical areas. For instance, Kanai et al. (2008) showed that the stimulation of the occipital cortex induced frequency-dependent phosphenes during light (at $\beta$ band) and dark (at $\alpha$ band). In a later study, Kanai et al. (2010) also demonstrated that the stimulation of the occipital cortex lowered the phosphene threshold induced by TMS. Feurra et al. (2011) provided further evidence of the effects of tACS on neocortical areas. In their study, tACS at $\alpha$ and $\gamma$ bands over the associative sensory cortex induced positive sensory sensations. It has also been demonstrated that entrainment with tACS at prefrontal sites during sleep improved procedural memory consolidation (Marshall et al., 2006).

No aftereffects of tACS were detected on motor cortex excitability. This has also been observed for the visual and sensory systems (Kanai et al., 2010; Feurra et al., 2011). This resembles a resonance-like effect, in analogy with a guitar string, which starts and ends to vibrate only until the closest string produces a sound. A physiological hypothesis is that the frequency-dependent corticospinal facilitation might have been induced by a tACS-driven increase of synchronization at the natural frequency (i.e., $20 \mathrm{~Hz}$ ) of the resting stimulated motor cortex.

This synchronization may result from a potentiation of inhibitory processes acting on quiescent motoneurons, a neurophysiological mechanism already known to mediate recurrent Renshaw inhibition at a spinal level (Mattei et al., 2003). At the cortical level, this phenomenon may occur with different-but not mutually exclusive-modalities. For instance, the potentiation may act upon common inhibitory synaptic inputs distributed to synchronous discharging neurons (Moore et al., 1970). Alternatively, oscillatory phenomena or slow waves may interact and simultaneously affect the neuronal membrane potential through inhibitory synaptic inputs or nonsynaptic interactions 
(Jefferys, 1995; Desmaisons et al., 1999). Finally, tACS at $20 \mathrm{~Hz}$ may have reinforced emergent network oscillations from reverberating inhibitory loops, which usually oscillate within the $\beta$ range (Whittington et al., 2000). Undoubtedly, oscillatory phenomena lead to rhythmic synchronization, regardless of whether they affect the neuronal membrane potential or the neural network of the stimulated area. Additional plastic adaptations may explain tACS aftereffects following longer stimulation periods (Moliadze et al., 2010). These effects were not evident here, probably due to the brief application of tACS.

However, the functional relevance of such a regional $20 \mathrm{~Hz}$ oscillatory activity on motor control is complex and not fully understood yet: despite the execution, preparation or imagery of a movement desynchronizes the $20 \mathrm{~Hz}$ activity in absolute terms (Jasper and Penfield, 1949; Chatrian et al., 1959; Pfurtscheller and Aranibar, 1977; Salmelin and Hari, 1994; Rossi et al., 2002), there is a relative increase of the coherence in the $\beta$ range between scalp-recorded EEG and EMG activity of the engaged muscles during actual movements (Kilner et al., 1999; Salenius and Hari, 2003; Kristeva et al., 2007). Moreover, whereas the entrainment at $20 \mathrm{~Hz}$ by tACS applied on the motor cortex increases corticomuscular coherence, some kinematic details of the movements are slower under the same circumstances (Pogosyan et al., 2009).

The current results provide a key to reconcile the apparently discordant findings of tACS-induced increase of corticospinal output with the interference on motor performance. It can be hypothesized that the increased corticospinal output triggered by the $20 \mathrm{~Hz}$ tACS-induced entrainment of the natural frequency of the resting motor areas probably takes place at the expense of the selectivity of motoneuronal recruitment. Several studies suggest that the majority of neurons in the motor cortex exhibit an intrinsic tendency to fire at $\beta$ range (Wetmore and Baker, 2004; Chen and Fetz, 2005). This mechanism could be amplified when neurons are externally tuned by tACS, according to the resonance-like hypothesis. In this vein, tACS at $\beta$ range may increase the likelihood that fast-conducting pyramidal neurons fire after each TMS pulse. Similarly, an increase of corticomuscular coherence during movements, paralleled by worsening of the motor performance (Pogosyan et al., 2009), might indicate a wider than necessary motoneural recruitment, as happens in the case of a movement disorder such as Parkinson's disease (Hammond et al., 2007).

The increase of corticospinal output induced by tACS at $\beta$ range is consistent among subjects, and it seems to be potent enough to overcome the poor relationships between amplitude/ phase of oscillatory $20 \mathrm{~Hz}$ spontaneous activity of rolandic neurons and the size of motor responses evoked by TMS of the motor cortex (Zarkowski et al., 2006; Mitchell et al., 2007; Lepage et al., 2008; Sauseng et al., 2009). A likely explanation is that spontaneous oscillations and MEPs size reflect the excitability of the human motor system in overlapping, but not identical, neuronal populations (Mäki and Ilmoniemi, 2010). According to the resonance-like hypothesis, tACS may have tuned these neuronal populations simultaneously, again making them more prone to fire after the TMS pulse. Only future and technologically challenging studies that combine online EEG and tACS with TMS will definitely confirm or reject the physiological hypotheses that are postulated here.

In summary, current findings represent the first attempt to directly quantify the corticospinal output in living humans during tACS applied at a physiological range on the motor system. The strict frequency dependence and regional specificity of tACS effects make this approach a potentially useful tool to be applied in the investigation of motor output dysfunctions, like Parkinson's disease or other movement disorders. Moreover, they may offer an opportunity to improve communication in Brain Computer Interface applications based on sensorimotor EEG rhythms (McFarland et al., 2000).

\section{References}

Adrian ED, Moruzzi G (1939) Impulses in the pyramidal tract. J Physiol 97:153-199.

Antal A, Boros K, Poreisz C, Chaieb L, Terney D, Paulus W (2008) Comparatively weak after-effects of transcranial alternating current stimulation (tACS) on cortical excitability in humans. Brain Stimul 1:97-105.

Baker SN (2007) Oscillatory interactions between sensorimotor cortex and the periphery. Curr Opin Neurobiol 17:649-655.

Bikson M, Datta A, Rahman A, Scaturro J (2010) Electrode montages for tDCS and weak transcranial electrical stimulation: role of "return" electrode's position and size. Clin Neurophysiol 121:1976-1978.

Buzsáki G, Draguhn A (2004) Neuronal oscillations in cortical networks. Science 304:1926-1929.

Chatrian GE, Petersen MC, Lazarte JA (1959) The blocking of the rolandic wicket rhythm and some central changes related to movement. Electroencephalogr Clin Neurophysiol 11:497-510.

Chen D, Fetz EE (2005) Characteristic membrane potential trajectories in primate sensorimotor cortex neurons recorded in vivo. J Neurophysiol 94:2713-2725.

Cincotta M, Giovannelli F, Borgheresi A, Balestrieri F, Toscani L, Zaccara G, Carducci F, Viggiano MP, Rossi S (2010) Optically tracked neuronavigation increases the stability of hand-held focal coil positioning: evidence from "transcranial" magnetic stimulation-induced electrical field measurements. Brain Stimul 3:119-123.

Datta A, Bansal V, Diaz J, Patel J, Reato D, Bikson M (2009) Gyri-precise head model of transcranial direct current stimulation: improved spatial focality using a ring electrode versus conventional rectangular pad. Brain Stimul 2:201-207.

Desmaisons D, Vincent JD, Lledo PM (1999) Control of action potential timing by intrinsic subthreshold oscillations in olfactory bulb output neurons. J Neurosci 19:10727-10737.

Di Lazzaro V, Oliviero A, Pilato F, Mazzone P, Insola A, Ranieri F, Tonali PA (2003) Corticospinal volleys evoked by transcranial stimulation of the brain in conscious humans. Neurol Res 25:143-150.

Engel AK, Fries P, Singer W (2001) Dynamic predictions: oscillations and synchrony in top-down processing. Nat Rev Neurosci 2:704-716.

Epsztein J, Lee AK, Chorev E, Brecht M (2010) Impact of spikelets on hippocampal CA1 pyramidal cell activity during spatial exploration. Science 327:474-477.

Feurra M, Paulus W, Walsh V, Kanai R (2011) Frequency specific modulation of human somatosensory cortex. Front Psychol 2:13.

Gastaut H (1952) Etude electrocorticographique de la reactivite des rhytmes rolandiques. Rev Neurol 87:176-182.

Gray CM, König P, Engel AK, Singer W (1989) Oscillatory responses in cat visual cortex exhibit inter-columnar synchronization which reflects global stimulus properties. Nature 338:334-337.

Hallett M (2000) Transcranial magnetic stimulation and the human brain. Nature 406:147-150.

Hammond C, Bergman H, Brown P (2007) Pathological synchronization in Parkinson's disease: networks, models and treatments. Trends Neurosci 30:357-364

Henneman E, Clamann HP, Gillies JD, Skinner RD (1974) Rank order of motoneurons within a pool: law of combination. J Neurophysiol $37: 1338-1349$.

Jasper H, Penfield W (1949) Electrocorticograms in man: effect of voluntary movement upon the electrical activity of the precentral gyrus. Eur Arch Psychiatry Clin Neurosci 183:163-174.

Jefferys JG (1995) Nonsynaptic modulation of neuronal activity in the brain: electric currents and extracellular ions. Physiol Rev 75:689-723.

Kanai R, Chaieb L, Antal A, Walsh V, Paulus W (2008) Frequencydependent electrical stimulation of the visual cortex. Curr Biol 18:1839-1843.

Kanai R, Paulus W, Walsh V (2010) Transcranial alternating current stimulation (tACS) modulates cortical excitability as assessed by TMSinduced phosphene thresholds. Clin Neurophysiol 121:1551-1554.

Kilner JM, Baker SN, Salenius S, Jousmäki V, Hari R, Lemon RN (1999) 
Task-dependent modulation of $15-30 \mathrm{~Hz}$ coherence between rectified EMGs from human hand and forearm muscles. J Physiol 516:559-570.

Koch G, Ruge D, Cheeran B, Fernandez Del Olmo M, Pecchioli C, Marconi B, Versace V, Lo Gerfo E, Torriero S, Oliveri M, Caltagirone C, Rothwell JC (2009) TMS activation of interhemispheric pathways between the posterior parietal cortex and the contralateral motor cortex. J Physiol 587:4281-4292.

Kristeva R, Patino L, Omlor W (2007) Beta-range cortical motor spectral power and corticomuscular coherence as a mechanism for effective corticospinal interaction during steady-state motor output. Neuroimage 36:785-792.

Kuhlman WN (1978) Functional topography of the human mu rhythm. Electroencephalogr Clin Neurophysiol 44:83-93.

Lepage JF, Saint-Amour D, Théoret H (2008) EEG and neuronavigated single-pulse TMS in the study of the observation/execution matching system: are both techniques measuring the same process? J Neurosci Methods 175:17-24.

Liddell EG, Phillips CG (1952) The cortical representation of motor units. Brain 75:510-525.

Llinás R, Ribary U (1993) Coherent 40-Hz oscillation characterizes dream state in humans. Proc Natl Acad Sci U S A 90:2078-2081.

Mäki H, Ilmoniemi RJ (2010) EEG oscillations and magnetically evoked motor potentials reflect motor system excitability in overlapping neuronal populations. Clin Neurophysiol 121:492-501.

Marshall L, Helgadóttir H, Mölle M, Born J (2006) Boosting slow oscillations during sleep potentiates memory. Nature 444:610-613.

Mattei B, Schmied A, Mazzocchio R, Decchi B, Rossi A, Vedel JP (2003) Pharmacologically induced enhancement of recurrent inhibition in humans: effects on motoneurone discharge patterns. J Physiol 548:615-629.

McFarland DJ, Miner LA, Vaughan TM, Wolpaw JR (2000) Mu and beta rhythm topographies during motor imagery and actual movements. Brain Topogr 12:177-186.

Miranda PC, Lomarev M, Hallett M (2006) Modeling the current distribution during transcranial direct current stimulation. Clin Neurophysiol 117:1623-1629.

Miranda PC, Faria P, Hallett M (2009) What does the ratio of injected current to electrode area tell us about current density in the brain during tDCS? Clin Neurophysiology 120:1183-1187.

Mitchell WK, Baker MR, Baker SN (2007) Muscle responses to transcranial stimulation in man depend on background oscillatory activity. J Physiol 583:567-579.

Moliadze V, Antal A, Paulus W (2010) Boosting brain excitability by transcranial high frequency stimulation in the ripple range. J Physiol 588:4891-4904.

Moore GP, Segundo JP, Perkel DH, Levitan H (1970) Statistical signs of synaptic interaction in neurons. Biophys J 10:876-900.

Niedermeyer E (1997) Alpha rhythms as normal and abnormal phenomena. Int J Psychophysiol 26:31-49.

Niedermeyer E (1999) The normal EEG of the waking adult. In: Electroencephalography: basic principles, clinical applications and related fields (Niedermeyer E, Lopes da Silva F, eds), pp 149-173. Baltimore: Lippincott, Williams \& Wilkins.

Nielsen JF (1996) Improvement of amplitude variability of motor evoked potentials in multiple sclerosis patients and in healthy subjects. Electroencephalogr Clin Neurophysiol 101:404-411.

Paulus W (2010) On the difficulties of separating retinal from cortical origins of phosphenes when using transcranial alternating current stimulation (tACS). Clin Neurophysiol 121:987-991.

Pfurtscheller G (1989) Functional topography during sensorimotor activa- tion studied with event-related desynchronization. J Clin Neurophysiol 6:75-84.

Pfurtscheller G (1998) EEG event-related desynchronization (ERD) and event-related synchronization (ERS). In: Electroencephalography: basic principles, clinical applications and related fields, Ed 4 (Niedermeyer E, Lopes da Silva FH, eds), pp 958-967, Baltimore: Lippincott, Williams \& Wilkins.

Pfurtscheller G, Aranibar A (1977) Event-related cortical desynchronization detected by power measurements of scalp EEG. Electroencephalogr Clin Neurophysiol 42:817-826.

Pogosyan A, Gaynor LD, Eusebio A, Brown P (2009) Boosting cortical activity at beta-band frequencies slows movement in humans. Curr Biol 19:1637-1641.

Pfurtscheller G, Stancák A Jr, Edlinger G (1997) On the existence of different types of beta rhythms below $30 \mathrm{~Hz}$. Electroencephalogr Clin Neurophysiol 102:316-325.

Rizzolatti G, Luppino G (2001) The cortical motor system. Neuron 31: 889-901.

Rosanova M, Casali A, Bellina V, Resta F, Mariotti M, Massimini M (2009) Natural frequencies of human corticothalamic circuits. J Neurosci 29:7679-7685.

Rossi S, Tecchio F, Pasqualetti P, Ulivelli M, Pizzella V, Romani GL, Passero S, Battistini N, Rossini PM (2002) Somatosensory processing during movement observation in humans. Clin Neurophysiol 113:16-24.

Rossi S, Hallett M, Rossini PM, Pascual-Leone A (2009) Safety, ethical considerations, and application guidelines for the use of transcranial magnetic stimulation in clinical practice and research. Clin Neurophysiol 120:2008-2039.

Rossini PM, Rossi S (2007) Transcranial magnetic stimulation: diagnostic, therapeutic, and research potential. Neurology 68:484-488.

Salenius S, Hari R (2003) Synchronous cortical oscillatory activity during motor action. Curr Opin Neurobiol 13:678-684.

Salmelin R, Hari R (1994) Characterization of spontaneous MEG rhythms in healthy adults. Electroencephalogr Clin Neurophysiol 91:237-248.

Sanes JN, Donoghue JP (1993) Oscillations in local field potentials of the primate motor cortex during voluntary movement. Proc Natl Acad Sci U S A 90:4470-4474.

Sauseng P, Klimesch W, Gerloff C, Hummel FC (2009) Spontaneous locally restricted EEG alpha activity determines cortical excitability in the motor cortex. Neuropsychologia 47:284-288.

Terney D, Chaieb L, Moliadze V, Antal A, Paulus W (2008) Increasing human brain excitability by transcranial high-frequency random noise stimulation. J Neurosci 28:14147-14155.

Thut G, Miniussi C (2009) New insights into rhythmic brain activity from TMS-EEG studies. Trends Cogn Sci 13:182-189.

Varela F, Lachaux JP, Rodriguez E, Martinerie J (2001) The brainweb: phase synchronization and large-scale integration. Nat Rev Neurosci 2: 229-239.

Wetmore DZ, Baker SN (2004) Post-spike distance-to-threshold trajectories of neurones in monkey motor cortex. J Physiol 555:831-850.

Whittington MA, Traub RD, Kopell N, Ermentrout B, Buhl EH (2000) Inhibition-based rhythms: experimental and mathematical observations on network dynamics. Int J Psychophysiol 38:315-336.

Zarkowski P, Shin CJ, Dang T, Russo J, Avery D (2006) EEG and the variance of motor evoked potential amplitude. Clin EEG Neurosci $37: 247-251$

Zarola F, Caramia MD, Paradiso C, Mariorenzi R, Martino G, Traversa R, Rossini PM (1989) Single fibre motor evoked potentials to brain, spinal roots and nerve stimulation. Comparisons of the 'central' and 'peripheral' response jitter to magnetic and electric stimuli. Brain Res 495:217-224. 